This document is confidential and is proprietary to the American Chemical Society and its authors. Do not copy or disclose without written permission. If you have received this item in error, notify the sender and delete all copies.

\title{
Voltage-driven conformational switching with distinct Raman signature in a single-molecule junction
}

\begin{tabular}{|r|l|}
\hline Journal: & Journal of the American Chemical Society \\
\hline Manuscript ID & ja-2017-12818w.R2 \\
\hline Manuscript Type: & Article \\
\hline Date Submitted by the Author: & 25-Feb-2018 \\
\hline & $\begin{array}{l}\text { Bi, Hai; Technische Universität München, Physik-Department } \\
\text { Palma, Carlos-Andres; Institute of Physics, Chinese Academy of Sciences, } \\
\text { Gong, Yuxiang; Technische Universität München, Physik-Department } \\
\text { Hasch, Peter; Technische Universität München, Physik-Department } \\
\text { Elbing, Mark; Fachhochschule Lubeck, } \\
\text { Mayor, Marcel; University of Basel, Department of Chemistry } \\
\text { Reichert, Joachim; TUM, Physik Department E20 } \\
\text { Barth, Johannes; TU München, Physics }\end{array}$ \\
\hline & \multicolumn{2}{|l}{} \\
\hline
\end{tabular}

\section{SCHOLARONE" \\ Manuscripts}




\title{
Voltage-driven conformational switching with distinct Raman signature in a single-molecule junction
}

\author{
Hai $\mathrm{Bi}^{1+}$, Carlos-Andres Palma ${ }^{1,2+}$, Yuxiang Gong ${ }^{1+}$, Peter Hasch ${ }^{1}$, Mark Elbing ${ }^{3}$, Marcel Mayor ${ }^{4,5}$, \\ Joachim Reichert*1 and Johannes V. Barth ${ }^{1}$ \\ ${ }^{1}$ Physics Department E20, Technical University of Munich, James-Franck-Str. 1, 85748 Garching, Germany \\ ${ }^{2}$ Institute of Physics, Chinese Academy of Sciences, 100190 Beijing, P.R. China \\ ${ }^{3}$ Department of Applied Natural Sciences, Lübeck University of Applied Sciences, Mönkhofer Weg 239, 23562 Lübeck, Ger- \\ many \\ ${ }^{4}$ Institute of Nanotechnology (INT), Karlsruhe Institute of Technology (KIT), Hermann-von-Helmholtz-Platz 1, 76344 Karls- \\ ruhe, Germany \\ ${ }^{5}$ Department of Chemistry, University of Basel, St Johannsring 19, CH-4056 Basel, Switzerland
}

KEYWORDS Molecular junction spectroscopy, Raman, tip, molecular switch, DFT, molecular electronics, terphenyl.

\begin{abstract}
Precisely controlling well-defined, stable single-molecule junctions represents a pillar of single-molecule electronics. Early attempts to establish computing with molecular switching arrays were partly challenged by limitations in the direct chemical characterization of metal-molecule-metal junctions. While cryogenic scanning probe studies have advanced the mechanistic understanding of current and voltage induced conformational switching, metal-molecule-metal conformations are still largely inferred from indirect evidence. Hence, the development of robust, chemically-sensitive techniques is instrumental for the advancement in the field. Here we probe the conformation of an engineered two-state molecular switch with vibrational spectroscopy, while simultaneously switching it by means of the applied voltage in a metalmolecule-metal junction. Our study emphasizes measurements of single-molecule Raman spectra in a room temperature stable single-molecule switch presenting a signal modulation of nearly two orders of magnitude.
\end{abstract}

Introduction. Performing logic operations with molecules is a frontier in computation ${ }^{1-2}$. Many strategies have been proposed toward this goal ${ }^{3}$ based on bi- or multistable switching. Prospective engineering methods in molecular computing are often centered on the electronic

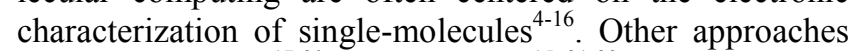
focus on chemical ${ }^{17-20}$ or mechanical ${ }^{15,21-23}$ studies. Investigations involving light-driven switches regularly employ photochromic molecules, such as azobenzenes ${ }^{21,24-27}$ or diarylethenes $^{28-32}$ to mention a few. Light also allows for complementary characterization and read-out of molecular states, exceeding present technology in terms of speed s3-34 $^{33}$ and power efficiency (by exploiting minimal electroninduced structural reorganization ${ }^{35}$ ). While light stands as a versatile read-out method, its implementation in metalmolecule-metal junctions remains challenging. Early attempts employed large area illumination of break junctions ${ }^{36-37}$ and tips ${ }^{11}$. To accelerate progress in molecular-scale computing, both optical and electrical read-out and switching must be demonstrated with stable, controlled single-molecule addressing. Here we report on voltagedriven conformational switching in covalently linked metal-molecule-metal junctions of substituted oligoterphenyls, where control over switching and read-out is demonstrated by employing a tetragonal tip serving as an electrode and waveguide. Exploiting Raman selection rules through physico-chemical design, we show how voltage-driven conformational changes enable Raman intensity modulation by nearly two orders of magnitude. Specifically, we remove the ground state polarizability and symmetry of a $p$ terphenyl-4,4"'-dithiol (2) molecule by employing the $2,2^{\prime}, 5^{\prime}, 2^{\prime \prime}$-tetramethylated (1) analogue ${ }^{38}$. With the highly sterically hindered, non-planar 1, the Raman signature is strongly suppressed. At high applied biases, the junctions can be modelled as transiently oxidized, and thus, extended $\pi$-conjugation, polarizability and Raman response are recovered (Fig. 1).

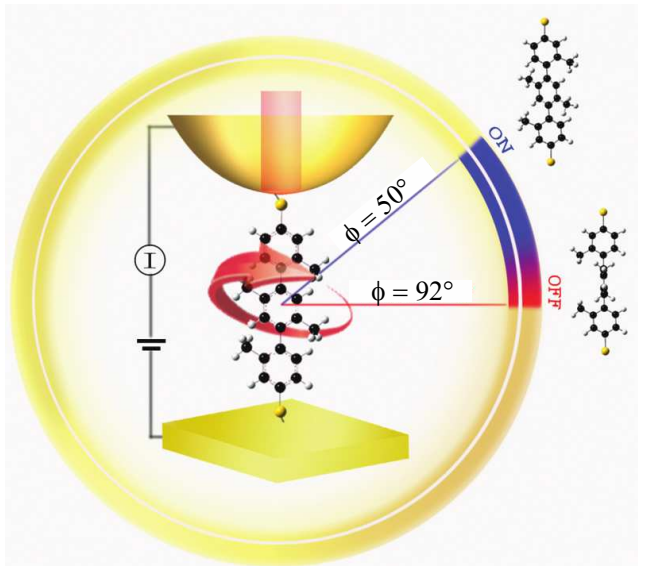

Figure 1. Voltage-driven Raman switching in a molecular junction spectroscopy setup Schematics showing conformational switching in a tip-molecule-substrate junction with Raman readout by optically addressing the molecules with a laser from the back of the tip. 


\section{Experimental details.}

Molecular junction spectroscopy. Molecular junction spectroscopy (MJS, Fig. 1) allows for simultaneous current-voltage $(I-V)$ and optical spectroscopy measurements in single molecules contacted by atomically-sharp electrodes. The thiol-terminated molecules are anchored to a gold film and contacted from the other side by the apex of a gold-covered $(20 \mathrm{~nm})$ tetrahedral glass (T-tip) fragment ${ }^{39-}$ 40. The tip-sample distance is controlled by a piezo. The technique integrates a Raman spectroscopy setup (Fig. S1) where the laser light (He-Ne laser, $12 \mathrm{~mW}, 632.8 \mathrm{~nm}$ ) is coupled through the glass body of the tetrahedral glass fragment into the backside of the apex of the tip. The tip acts as the light source, counter-electrode and light collector at the same time (Fig. 1). All measurements shown in the main text are recorded in high vacuum $\left(\sim 1 \times 10^{-7} \mathrm{mbar}\right)$.

Substrate preparation. The fabrication process of the glass substrate is the same as for the T-tip. The glass slide is coated with a $200-500 \mathrm{~nm}$ gold film. A droplet of the molecular solution $(0.5 \mathrm{mM})$ of 1 ( $\operatorname{ref}^{38}$ ) or $\mathbf{2}$ (Aldrich) in tetrahydrofuran (THF, Aldrich) is deposited with a pipette on the gold covered substrate. Then the substrate is put in an atmosphere saturated with the THF solvent for three hours which affords a dilute layer with the molecules covalently anchored to the substrate.

Molecular junction preparation. The substrate is prepared ex situ and fixed on the sample holder in the vacuum chamber. The tip is approached mechanically into the working distance of the piezo crystal with the help of a tunneling current feedback loop. To establish a molecular junction, the sample is approached with $V_{\text {sample }}=0.5 \mathrm{~V}$ to the tip repeatedly until the current jumps out of tunneling, typically between $I=1 \times 10^{-9}$ and $1 \times 10^{-7}$ A. Current-voltage characteristic curves between -1.5 and $1.5 \mathrm{~V}$ are then obtained. The histogram for this procedure is shown in Fig. 2a,d. When stable $I-V$ curves are found, the Raman spectra are recorded.

Density Functional Theory. The simulated Raman spectra were calculated with the quantum chemical package Firefly $^{41}$. The molecular geometries were energetically optimized to an energy difference of 0.00001 Hartree. Hessian activities were obtained employing the exchange and correlation $\mathrm{PBE}^{42}$ (keyword PBE86 in Firefly) functional using a $6-311 \mathrm{G}$ basis set. The stationary state PBE geometry and Hessian of the molecule were used as an input for calculating the Raman spectra with the Firefly package, employing a global hybrid SOGGA $11^{43}$ correlation functional including an optimized percentage of Hartree-Fock exchange energy ("SOGGAX") together with a TZV $3 p 3 d$ basis set with diffuse $s$ functions. As a simple approximation for molecular grafting on gold, calculations were performed by increasing the isotopic mass of the $\mathrm{S}$ and $\mathrm{H}$ elements of the thiol terminal groups to one million amu. Although electrodes can influence the electronic state, our approximation reproduces the Raman spectra in fine detail (vide infra) while aiding the reproducibility of the calculations (avoiding variation in the choice of dispersion interactions and pseudopotentials for the electrodes). $R$-factors are defined as the ratio between the sum of the square modelexperimental differences and the sum of their squares.
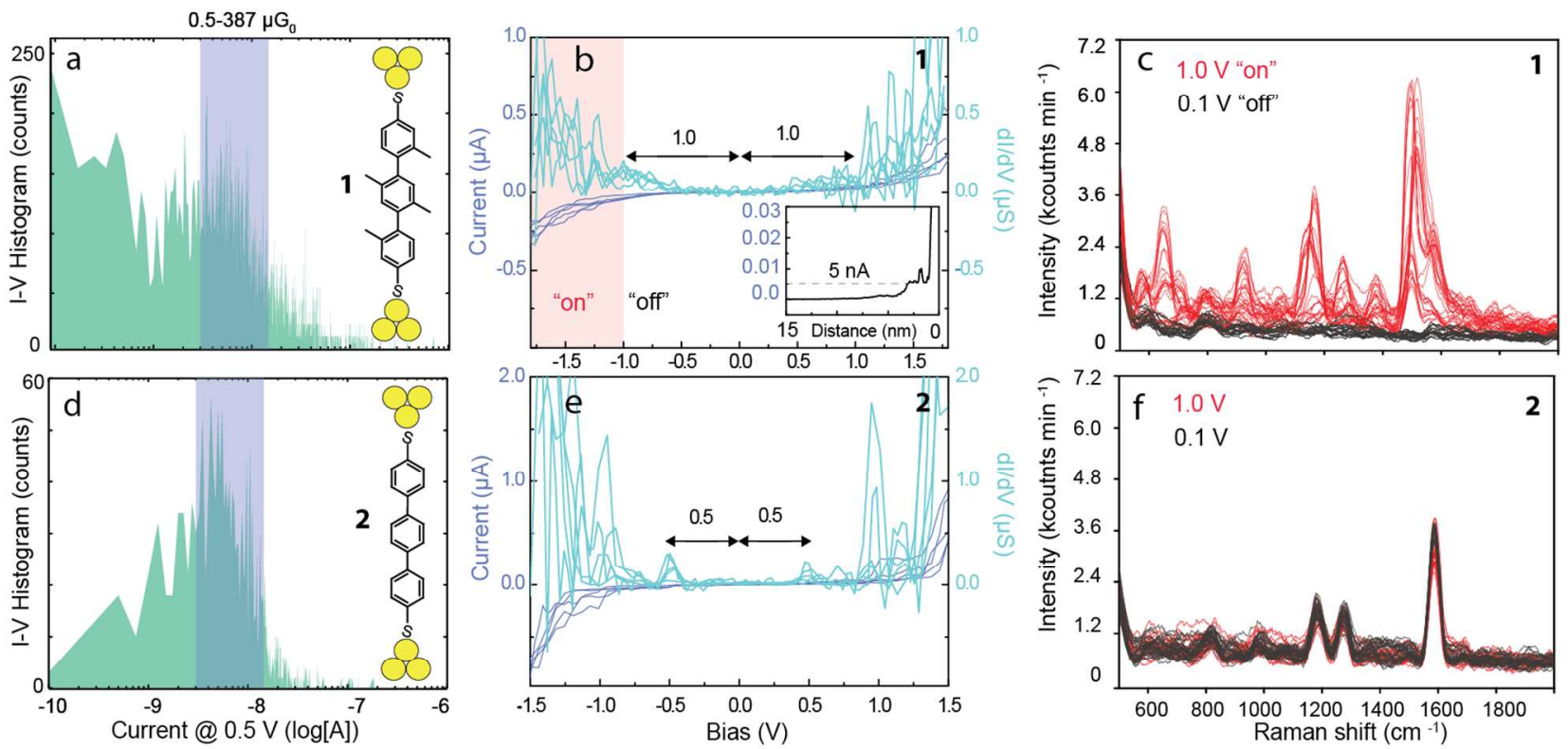

Figure 2. Voltage-driven Raman switching in a molecular junction spectroscopy setup. (a,d) Single-molecule current histogram curves, measured at $0.5 \mathrm{~V}$ while approaching a gold-covered glass tip to a substrate covered with $\mathbf{1}$ or $\mathbf{2}$. (b,e) Characteristic $I$ - $V$ curves (blue) extracted at single-molecule contact range between $0.5-387 \mu \mathrm{G}_{0}$ (blue in a,d) and corresponding derivatives $d I / d V$ (cyan). The raw data is shown unfiltered; the inset in (b) shows successful junction formation. (c,f) Raman signature of the pertinent junctions at biases of 1.0 and $0.1 \mathrm{~V}$, respectively. Only for 1 a clear on(red)-off(black) behavior is observed (excitation wave length $\lambda_{0}=632.8 \mathrm{~nm}$ ). 


\section{Results.}

Molecular junction Raman spectroscopy of 1 and 2. Upon preparation of a layer of thiol-anchored 1 on amorphous gold substrates, single molecules are contacted by the apertureless, gold-coated, near-field tip. Typical current histograms of 1 ( $\sim 600$, Fig. 2a) suggest a conductance between $0.5-387 \mu \mathrm{G}_{0}$ during single-molecule junction formation at $0.5 \mathrm{~V}$. The $I-V$ curves in this conductance regime are extracted, concomitantly with their respective Raman signals (Fig. 2b,c). Conductance windows are usually employed $^{10,44}$ to study only one metal-molecule-metal conformation and thereby reduce the occurrence of outlier Raman response ${ }^{45}$. In the selected regime, several junctions of $\mathbf{1}$ display broad transport channels appearing at a bias close to $\pm 1.0 \mathrm{~V}$ (Fig. 2b). At biases close to or exceeding the transport voltages, a clear Raman signal is observed (Fig. $2 \mathbf{c}$, red), featuring prominent peaks at approximately 650 , $930,1160,1270,1390,1490$ and $1580 \mathrm{~cm}^{-1}$. By contrast, $80 \%$ of the Raman signals recorded at $0.1 \mathrm{~V}$ fall below instrumental detection limits, i.e., the Raman signature is effectively suppressed. The observed voltage-driven switching can be repeatedly turned on and off at time scales limited by the junction stability only.

In order to put the intriguing switching behavior of $\mathbf{1}$ into perspective, we investigated the reference species 2 , i.e. the non-methylated analogue of $\mathbf{1}$. Figure 2d-e depicts the pertaining histogram along with extracted $I-V$ and $d I / d V$ curves for 2 . This time, the contact entails transport resonances starting at $0.5 \mathrm{~V}$. Moreover, in striking contrast to $\mathbf{1}$, the experimental Raman signature of $\mathbf{2}$ reproduced in Fig. 2f does not show any significant change in the Raman signature dependent on the junction voltage. Rather, the spectra display constant peaks near 1180,1275 and 1590 $\mathrm{cm}^{-1}$. Because both molecules are relatively apolar and neither linear nor quadratic shifts appear in the Raman spectra, the observed switching of the Raman signal of $\mathbf{1}$ is clearly indicative of distinct and reversible conformational changes, whereby Stark or related electric field effects reported elsewhere are excluded. ${ }^{46-49}$

Charge-transport induced conformational switching. Transient charging effects ${ }^{46,50}$ are a well-known point of departure for the elucidation of bias-induced conformational effects. In oligophenylenes, the removal/addition of one electron increases the $\pi$-conjugation along the oligophenylene backbone due to the formation of quinoids or semi-quinoids resonance structures ${ }^{51}$. Such transient charging events occur during hopping transport or under a strong polarization regime ${ }^{52}$. In the semi-quinoid form, bond orders between phenyl rings amount to one and two, and the phenylenes' conformation is semi-coplanar. In the quinoid form, bond orders are close to two, and conformation is close to coplanar. Thus, oligophenylenes ${ }^{53}$ generally tend toward planar $\pi$-conjugation upon reduction or oxidation $^{54}$. In this manner, a stationary point in the interphenyl torsion (IPT) potential energy surface (Fig. 3a, black line) of an oligophenylene such as $\mathbf{1}$ can be rationalized and further engineered through the interplay between the gradient of the attractive conjugation energy, which tends to planarize the structure, and the gradient of the steric repulsion. Accordingly, the neutral optimization of $\mathbf{1}$ at the PBE level of theory features dihedral, or IPT angles, of $\varphi_{1}=\varphi_{2}=92^{\circ}$. Conversely, the positively charged, singlet state of $\mathbf{1}+$ (Fig. 3a, red line) shows a geometrical stationary point with reduced IPT angles, $\varphi_{1}=\varphi_{2}=50^{\circ}$. Thus, planarization is maximum for the oxidized form $\mathbf{1 +}$, which might transiently occur during charge transport. Because of the large steric repulsion caused by the methyl substituents, 1+ cannot adopt a fully planar quinoid structure. The increased $\pi$-conjugation in this case is geometrically manifested in $\pi$-twisted orbitals (r.h.s. Fig. 3a).

The Raman activity of a molecule is dictated by the internal product of the polarizability tensor with the overlap between initial and final phonon wavefunctions. Extended $\pi$-conjugation allows for higher molecular polarizabilities due to wavefunction delocalization, and consequently, increased Raman signals. Therefore, by decreasing the mode's polarizability Raman activity can be turned "off" from an active "on" state. It is therefore expected that the Raman signature of $\mathbf{1}+$ decreases in its neutral form, where the $\pi$-conjugated character is at its lowest. The case is sensibly different for molecule $\mathbf{2}$. The ground state IPT of $\mathbf{2}$ $\left(25^{\circ}\right)$ is close to the oxidized form $\left(37^{\circ}\right)$. Thus, the Raman activity of $\mathbf{2}$ should remain similar between neutral and transiently charged $\mathbf{2}$.

Raman spectra comparison to statically charged DFT calculations. Further proof for a transient charging mechanism is provided through Raman spectrum analysis. First, experimental Raman spectra (Fig. 3b-i) are chosen from the molecular junction Raman survey in Fig. 2c,f. From various calculated oxidation states and DFT functionals (Supporting Fig. S2,S3), the experimental Raman spectrum at $1.0 \mathrm{~V}$ (Fig. 3b-e) correlates best $(R=0.27)$ to the DFT-simulated Raman spectra of positively charged 1+ species. This suggests that charge (hole) transport in the molecule happens through a (partially) depleted HOMO. Switching to a seemingly formal $\mathbf{1}+$ charged state could imply that electron depopulation is much faster than the electron current repopulation times. For the neutral state of $\mathbf{1}$, the Raman activity of the $\sim 1600 \mathrm{~cm}^{-1}$ mode falls below $1000 \mathrm{~A}^{4} \mathrm{amu}^{-1}$, or 500 -fold less than the computed charged 1+ species (Figs. 3e), in agreement with the experimental result.

To investigate the role of $\pi$-conjugation in "switching on" the Raman activity, we performed DFT calculations employing the Hessian of the conjugated conformation $\left(\varphi_{1}\right.$ $\left.=50.8^{\circ}\right) \mathbf{1}+$ but the polarizability for the non-conjugated conformation $\left(\varphi_{1}=92.7^{\circ}\right)$. In this case, the $\sim 1600 \mathrm{~cm}^{-1}$ Raman signal of the so-calculated hybrid positively charged molecule drops only by a factor of 30 (instead of 500), to less than $10000 \mathrm{~A}^{4} \mathrm{amu}^{-1}$. These observations and comparisons indicate that increased polarizability via extended $\pi$-conjugation is decisive for the switchable Raman activity of $\mathbf{1}$. 

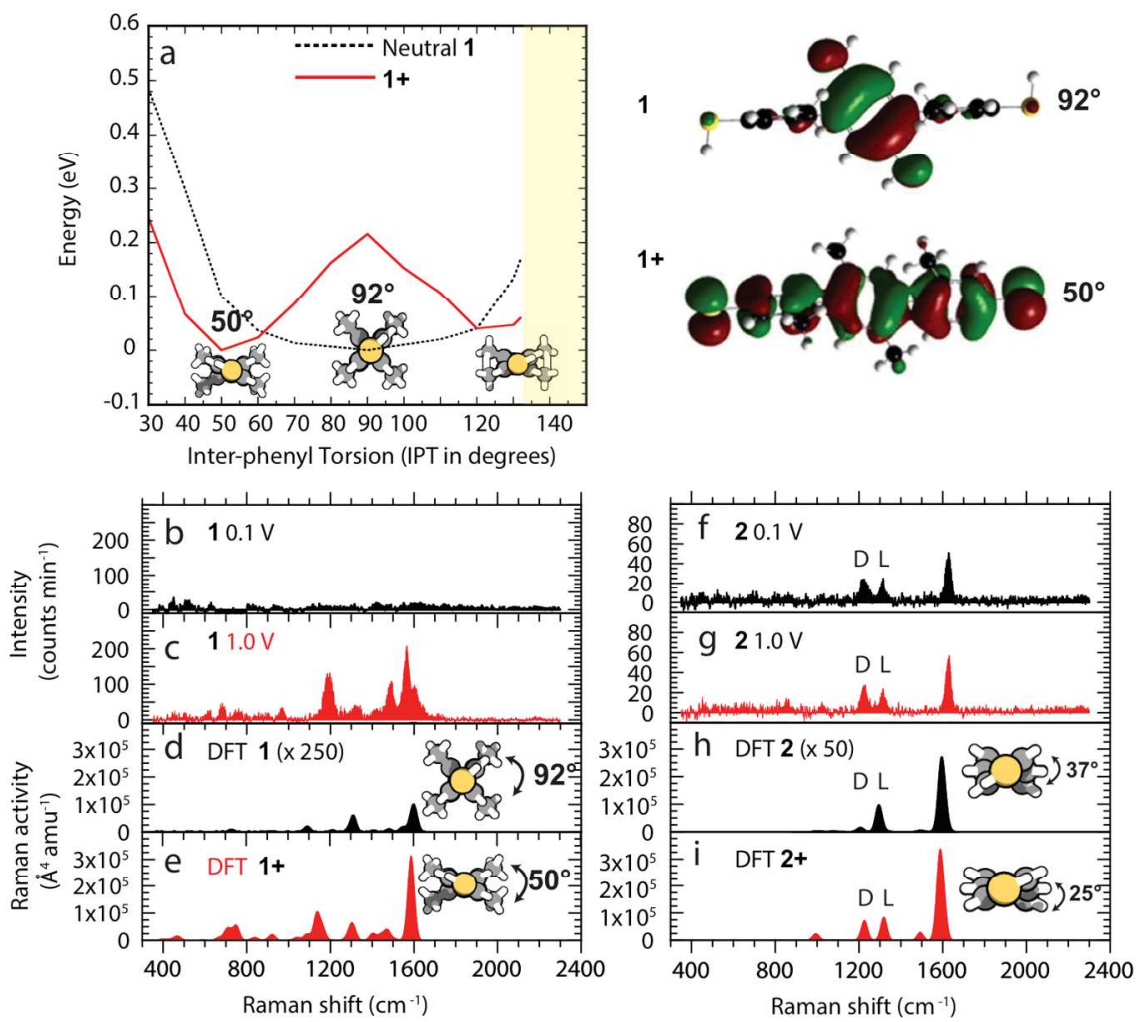

Figure 3. Modeling transient charging via static charges in DFT. (a) Density Functional Theory (DFT) energy comparison along the interphenyl torsion (IPT) of the central ring of $\mathbf{1}$ and $\mathbf{1}^{+}$. The area where methyl units of adjacent phenylenes overlap is indicated in yellow. The HOMO of neutral $\mathbf{1}$ and HOMO of $\mathbf{1}+$, showing $\pi$-conjugation through overlap of orbitals with equal parity are also shown on the right hand side. Typical spectra of $\mathbf{1}$ at (b) 0.1 ("off") and (c) $1.0 \mathrm{~V}$ ("on"). Scaled DFT Raman spectra of (d) neutral ("off") 1 times a factor of 250 and (e) charged ("on") 1+ singlet state. Typical spectra of $\mathbf{2}$ at (f) 0.1 and (g) 1.0 V. Scaled DFT Raman spectra of (h) 2 (times a factor of 50) and (i) $2+$ singlet state. The insets are representations of the optimized molecular structures with perspectives looking along the molecules' axes in order to visualize the IPT angles.

Indeed, the calculated Raman spectra of $\mathbf{2}$ (Fig. $\mathbf{3 f}-\mathbf{i})$ is not influenced significantly by its charge state. Supporting Figure S3 exhaustively compares the experimental spectra to the calculated spectra for different electronic states: singlet +2 , singlet +1 , neutral, singlet -1 and singlet -2 (calculated spectra are automatically offset to best fit the theory for ease of comparison).

Finally, no anti-Stokes Raman signal was detected during HOMO charge transport for neither $\mathbf{2}+$ nor $\mathbf{1}+$. One explanation for the absence of a significant anti-Stokes signal in the 600 to $2000 \mathrm{~cm}^{-1}$ region is the high resistance of 1 and 2. Lower currents imply that current-driven excitations of vibrational states are smaller and therefore below detection limits. The studies with $\mathbf{1}$ and $\mathbf{2}$ thus demonstrate that a Raman on/off switch is fundamentally accomplished by strongly increasing/decreasing the polarizability of one state, since symmetry selection rules for both charged and neutral IPT geometries are similar.

Cryogenic molecular junction Raman spectroscopy. From the analysis presented above, it follows that $\mathbf{2}$ should retain Raman activity without the necessity of transient charging. The Raman spectrum of $\mathbf{2}$ also provides important insight into a freely rotating IPT barrier contributing to the Raman activity. Starting from the experimental signature at 1220 and $1280 \mathrm{~cm}^{-1}$ (so-called $D$ and $L$ bands, respectively) both experimental spectra of 2 show a $D / L$ ratio of one, characteristic of co-planar $\left(\mathrm{IPT}=0^{\circ}\right)$ or librat- ing oligophenylenes ${ }^{55}$. This indicates that IPT angle librations occur at room temperature (Fig. 3b,c). Indeed, DFT predicts similar IPTs of $25^{\circ}$ and $37^{\circ}$ between the charged and neutral state of $\mathbf{2}$. Freezing room temperature librations may allow bias-dependent detection between the small, but noticeable differences in Raman activities predicted by DFT (Fig. 3h,i).

In order to substantiate this hypothesis, we performed cryogenic measurements at $77 \mathrm{~K}$, whereby we also monitored the systems' response over the entire bias sweeps (Fig. 4). The corresponding data sets identify again a switching behaviour of 1 with a turn-on voltage at $\sim 0.9 \mathrm{~V}$. For 2 , we detect a marked $\sim 1.5$-fold increase in the $\sim 1600$ $\mathrm{cm}^{-1}$ Raman signal peaking beyond $\pm 0.5 \mathrm{~V}$. This change is assigned to the different response of the neutral IPT $37^{\circ}$ and transiently oxidized IPT $25^{\circ}$ conformations at low temperatures. Evidently, the Raman signal of $\mathbf{2}$ cannot be turned off, since the unhindered ground state IPT $37^{\circ}$ remains polarizable (in comparison, the polarizable "on" state of $\mathbf{1}$ is achieved at the theoretically predicted IPT of $\left.50^{\circ}\right)$. 

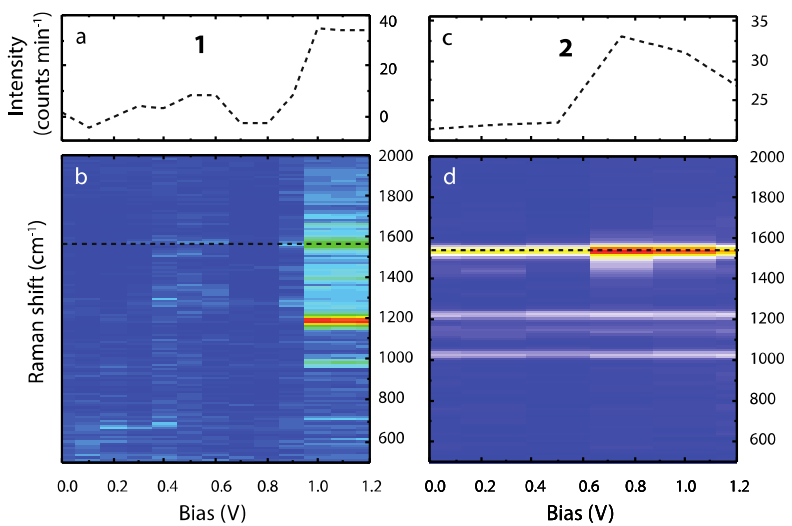

Figure 4. On/off Raman behavior of 1 and 2 at croygenic conditions $(\mathbf{T}=\mathbf{7 7} \mathbf{K})$. $(\mathbf{a}, \mathbf{b})$ The Raman signature of $\mathbf{1}$ displays switching characteristics similar to room temperature measurements. (c,d) For 2 now the Raman intensity of prominent states increases for bias exceeding $0.5 \mathrm{~V}$. Dashed lines in $(\mathrm{a}, \mathrm{c})$ emphasize the prominent line of the $\sim 1600 \mathrm{~cm}^{-1}$ Raman signal.

Summary. We have shown conformational switching controlled by voltage in a single-molecule junction. Comparative, low-temperature and theoretical studies served to experimentally identify alterations in the Raman spectrum with applied bias, as polarizability switching driven by $\pi$ conjugation, which can be tailored by molecular design. The possibility of addressing physico-chemical phenomena with single-molecule resolution shown here, serves as a departure point for a new generation of nanomechanical studies using MJS setups. Technologically, our investigations present single-molecule monitoring and manipulation with exquisite control, opening new avenues in multi-logic single-molecule computing.

\section{ASSOCIATED CONTENT}

\section{Supporting Information}

Additional details on experimental procedures. This material is available free of charge via the Internet at http://pubs.acs.org.

\section{AUTHOR INFORMATION}

\section{Corresponding Author}

*Email: joachim.reichert@ph.tum.de (J.R.)

tequally contributing authors

\section{Funding Sources}

We acknowledge the Deutsche Forschungsgemeinschaft (DFG) for financial support via the SPP 1234 (Grant RE2592), the Munich Centre for Advanced Photonics (MAP), the China Scholarship Council (CSC), the European Research Council via Advanced Grant MolArt (Grant 247299), the Institute of Physics and Frontier Research Grant of the Chinese Academy of Sciences, and the European Union's Horizon 2020 research and innovation programme as Future and Emerging Technologies Action (Grant 664878).

\section{ACKNOWLEDGMENT}

C.-A. Palma thanks R. Bader, M. Bopp and the LeibnizRechenzentrum (LRZ) for support. We thank Alexander Holleitner at the Zentrum für Nanotechnologie und Nanomaterialien for infrastructure support, and Anthoula C. Papageorgiou as well as Elke Scheer for discussions.

\section{ABBREVIATIONS}

MJS Molecular junction setup

\section{REFERENCES}

1. Reed, M. A.; Tour, J. M., Sci. Am. 2000, 282, 86-

93.

2. Markoff, J., Designing the Next Wave of

Computer Chips. The New York Times 2014.

3. de Silva, A. P.; Uchiyama, S., Nat. Nanotech.

2007, 2, 399-410.

4. $\quad$ Park, H.; Park, J.; Lim, A. K. L.; Anderson, E. H.; Alivisatos, A. P.; McEuen, P. L., Nature 2000, 407, 57-60. 5. Moresco, F.; Meyer, G.; Rieder, K. H.; Tang, H.; Gourdon, A.; Joachim, C., Phys. Rev. Lett. 2001, 86, 672675.

6. Donhauser, Z. J.; Mantooth, B. A.; Kelly, K. F.; Bumm, L. A.; Monnell, J. D.; Stapleton, J. J.; Price, D. W.; Rawlett, A. M.; Allara, D. L.; Tour, J. M.; Weiss, P. S., Science 2001, 292, 2303-2307.

7. Xu, B. Q.; Tao, N. J. J., Science 2003, 301, 12211223.

8. Kubatkin, S.; Danilov, A.; Hjort, M.; Cornil, J.; Brédas, J. L.; Stuhr-Hansen, N.; Hedegård, P.; Bjornhølm, T., Nature 2003, 425, 698-701.

9. Iancu, V.; Hla, S. W., Proc. Natl. Acad. Sci. U. S. A. 2006, 103, 13718-13721.

10. Venkataraman, L.; Klare, J. E.; Nuckolls, C.; Hybertsen, M. S.; Steigerwald, M. L., Nature 2006, 442, 904-907.

11. Liu, Z.; Ding, S. Y.; Chen, Z. B.; Wang, X.; Tian, J. H.; Anema, J. R.; Zhou, X. S.; Wu, D. Y.; Mao, B. W.; Xu, X.; Ren, B.; Tian, Z. Q., Nat. Commun. 2011, 2:305.

12. Kim, Y.; Pietsch, T.; Erbe, A.; Belzig, W.; Scheer, E., Nano Lett. 2011, 11, 3734-3738.

13. Bruot, C.; Hihath, J.; Tao, N. J., Nat. Nanotech. 2012, 7, 35-40.

14. Auwärter, W.; Seufert, K.; Bischoff, F.; Écija, D.; Vijayaraghavan, S.; Joshi, S.; Klappenberger, F.;

Samudrala, N.; Barth, J. V., Nat. Nanotech. 2012, 7, 41-46. 15. Su, T. A.; Li, H. X.; Steigerwald, M. L.;

Venkataraman, L.; Nuckolls, C., Nat. Chem. 2015, 7, 215 220.

16. Yelin, T.; Korytar, R.; Sukenik, N.; Vardimon, R.; Kumar, B.; Nuckolls, C.; Evers, F.; Tal, O., Nat. Mater. 2016, 15, 444-449.

17. Credi, A.; Balzani, V.; Langford, S. J.; Stoddart, J. F., J. Am. Chem. Soc. 1997, 119, 2679-2681.

18. de Silva, A. P.; McClenaghan, N. D., J. Am. Chem. Soc. 2000, 122, 3965-3966.

19. Margulies, D.; Melman, G.; Shanzer, A., J. Am. Chem. Soc. 2006, 128, 4865-4871.

20. Gupta, T.; van der Boom, M. E., Angew. Chem. Intl. Ed. 2008, 47, 5322-5326.

21. Hugel, T.; Holland, N. B.; Cattani, A.; Moroder, L.; Seitz, M.; Gaub, H. E., Science 2002, 296, 1103-1106. 
22. Brough, B.; Northrop, B. H.; Schmidt, J. J.; Tseng, H. R.; Houk, K. N.; Stoddart, J. F.; Ho, C. M., Proc. Nat. Acad. Sci. U.S.A. 2006, 103, 8583-8588.

23. Lussis, P.; Svaldo-Lanero, T.; Bertocco, A.; Fustin, C. A.; Leigh, D. A.; Duwez, A. S., Nat.

Nanotechnol. 2011, 6, 553-557.

24. Mativetsky, J. M.; Pace, G.; Elbing, M.; Rampi, M. A.; Mayor, M.; Samorì, P., J. Am. Chem. Soc. 2008, 130, 9192-9193.

25. Cao, Y.; Dong, S. H.; Liu, S.; Liu, Z. F.; Guo, X. F., Angew. Chem. Intl. Ed. 2013, 52, 3906-3910.

26. Kim, Y.; Garcia-Lekue, A.; Sysoiev, D.; Frederiksen, T.; Groth, U.; Scheer, E., Phys. Rev. Lett. 2012, 109, 226801.

27. Margapoti, E.; Li, J.; Ceylan, Ö.; Seifert, M.; Nisic, F.; Anh, T. L.; Meggendorfer, F.; Dragonetti, C.; Palma, C.-A.; Barth, J. V.; Finley, J. J., Adv. Mater. 2015, 27, 1426-1431.

28. Dulić, D.; van der Molen, S. J.; Kudernac, T.; Jonkman, H. T.; de Jong, J. J. D.; Bowden, T. N.; van Esch, J.; Feringa, B. L.; van Wees, B. J., Phys. Rev. Lett. 2003, 91, 207402.

29. Irie, M., Chem. Rev. 2000, 100, 1685-1716.

30. Kim, Y.; Hellmuth, T. J.; Sysoiev, D.; Pauly, F.; Pietsch, T.; Wolf, J.; Erbe, A.; Huhn, T.; Groth, U.; Steiner, U. E.; Scheer, E., Nano Lett. 2012, 12, 3736-3742.

31. Jia, C. C.; Wang, J. Y.; Yao, C. J.; Cao, Y.; Zhong, Y. W.; Liu, Z. R.; Liu, Z. F.; Guo, X. F., Angew. Chem. Intl. Ed. 2013, 52, 8666-8670.

32. Jia, C. C.; Migliore, A.; Xin, N.; Huang, S. Y.; Wang, J. Y.; Yang, Q.; Wang, S. P.; Chen, H. L.; Wang, D. M.; Feng, B. Y.; Liu, Z. R.; Zhang, G. Y.; Qu, D. H.; Tian, H.; Ratner, M. A.; Xu, H. Q.; Nitzan, A.; Guo, X. F., Science 2016, 352, 1443-1445.

33. Hosaka, K.; Shimada, H.; Chiba, H.; Katsuki, H.; Teranishi, Y.; Ohtsuki, Y.; Ohmori, K., Phys. Rev. Lett. 2010, 104, 180501.

34. Schiffrin, A.; Paasch-Colberg, T.; Karpowicz, N.; Apalkov, V.; Gerster, D.; Muhlbrandt, S.; Korbman, M.; Reichert, J.; Schultze, M.; Holzner, S.; Barth, J. V.; Kienberger, R.; Ernstorfer, R.; Yakovlev, V. S.; Stockman, M. I.; Krausz, F., Nature 2013, 493, 70-74.

35. Valkenier, H.; Guedon, C. M.; Markussen, T.; Thygesen, K. S.; van der Molen, S. J.; Hummelen, J. C., Phys. Chem. Chem. Phys. 2014, 16, 653-662.

36. Tian, J. H.; Liu, B.; Li, X. L.; Yang, Z. L.; Ren, B.; Wu, S. T.; Tao, N. J.; Tian, Z. Q., J Am Chem Soc 2006, 128, 14748-14749.

37. Ward, D. R.; Halas, N. J.; Ciszek, J. W.; Tour, J. M.; Wu, Y.; Nordlander, P.; Natelson, D., Nano Lett 2008, 8, 919-924.
38. Lörtscher, E.; Elbing, M.; Tschudy, M.; von Hänisch, C.; Weber, H. B.; Mayor, M.; Riel, H., ChemPhysChem 2008, 9, 2252-2258.

39. Koglin, J.; Fischer, U. C.; Fuchs, H., Phys. Rev. B 1997, 55, 7977-7984.

40. Fischer, U. C.; Koglin, J.; Fuchs, H., J. MicroscOxford 1994, 176, 231-237.

41. Alex A. Granovsky Firefly version 8, www http://classic.chem.msu.su/gran/firefly/index.html.

42. Perdew, J. P.; Burke, K.; Wang, Y., Phys. Rev. B 1996, 54, 16533-16539.

43. Peverati, R.; Truhlar, D. G., J. Chem. Phys. 2011, $135,191102$.

44. Komoto, Y.; Fujii, S.; Nakamura, H.; Tada, T.; Nishino, T.; Kiguchi, M., Sci. Rep. 2016, 6:26606

45. Benz, F.; Schmidt, M. K.; Dreismann, A.; Chikkaraddy, R.; Zhang, Y.; Demetriadou, A.; Carnegie, C.; Ohadi, H.; de Nijs, B.; Esteban, R.; Aizpurua, J.; Baumberg, J. J., Science 2016, 354, 726-729.

46. Li, Y.; Doak, P.; Kronik, L.; Neaton, J. B.; Natelson, D., Proc. Natl. Acad. Sci. U. S. A. 2014, 111, 1282-1287.

47. Li, Y. J.; Zolotavin, P.; Doak, P.; Kronik, L.; Neaton, J. B.; Natelson, D., Nano Lett. 2016, 16, 11041109.

48. Toccafondi, C.; Picardi, G.; Ossikovski, R., J. Phys. Chem. C 2016, 120, 18209-18219.

49. Kaneko, S.; Murai, D.; Marques-Gonzalez, S.; Nakamura, H.; Komoto, Y.; Fujii, S.; Nishino, T.; Ikeda, K.; Tsukagoshi, K.; Kiguchi, M., J. Amer. Chem. Soc 2016, 138, 1294-1300.

50. de Nijs, B.; Benz, F.; Barrow, S. J.; Sigle, D. O.; Chikkaraddy, R.; Palma, A.; Carnegie, C.; Kamp, M.;

Sundararaman, R.; Narang, P.; Scherman, O. A.; Baumberg, J. J., Nat. Commun. 2017, 8:994.

51. Zojer, E.; Cornil, J.; Leising, G.; Brédas, J. L., Phys. Rev. B. 1999, 59, 7957-7968.

52. Galperin, M.; Ratner, M. A.; Nitzan, A.; Troisi, A., Science 2008, 319, 1056-1060.

53. Baker, K. N.; Fratini, A. V.; Resch, T.; Knachel, H. C.; Adams, W. W.; Socci, E. P.; Farmer, B. L., Polymer 1993, 34, 1571-1587.

54. Palma, C.-A.; Joshi, S.; Hoh, T.; Écija, D.; Barth, J. V.; Auwarter, W., Nano Lett 2015, 15, 2242-2248.

55. Heimel, G.; Somitsch, D.; Knoll, P.; Brédas, J. L.; Zojer, E., J. Chem. Phys. 2005, 122, 114511. 


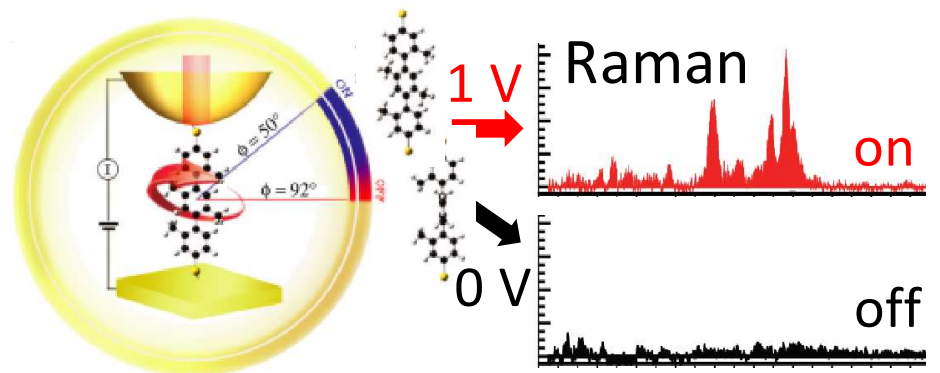

56

57 